\title{
COMMUNICATIONS
}

\section{Graphene prepared on SiC by chemical vapor deposition process at low temperature}

\author{
Petr Machac ${ }^{*}$
}

\begin{abstract}
Graphene preparation by the method of chemical vapour deposition on $\mathrm{SiC}$ substrates is described. Despite very low growth temperature $\left(1080^{\circ} \mathrm{C}\right)$ and with use of methane atmosphere, carbon layers in the form of multi-layer graphene were prepared. Graphene quality was verified by means of available analytical methods: Raman spectroscopy, X-ray photoelectron spectroscopy, Van der Paw method.
\end{abstract}

K e y w ords: graphene, CVD process, $\mathrm{SiC}$

\section{Introduction}

Graphene is a promising material that can be used in numerous applications, from electronics to medicine. For electronics, highly important is the direct possibility of preparation of graphene layers on semiconductor materials, such as silicon carbide. As a standard, the method of $\mathrm{SiC}$ thermal dissociation sometime also called the method of epitaxial growth [1] is being used. The principal disadvantage of this method is the necessity of reaching high temperature for graphene growth, ranging from $1300^{\circ} \mathrm{C}$ to $1600^{\circ} \mathrm{C}$. Certain decrease of the required temperature is offered by the method of chemical vapour deposition (CVD). Depending on the source of carbon, temperatures $900^{\circ} \mathrm{C}($ toluene - [2] $) ; 1200^{\circ} \mathrm{C}$ (ethylene - [3]); $1300^{\circ} \mathrm{C}$ (propane - [4]) were reached. In our work, the CVD apparatus nanoCVD-8G (Moorfield Nanotechnology Ltd) with cold-wall reactor was used; this apparatus utilizes methane as a carbon source and enables graphene growth up to temperature of $1100^{\circ} \mathrm{C}$.

\section{Experimental details}

As a substrate for graphene growth were used the $4 \mathrm{H}$ $\mathrm{SiC}$ plates, both from the $\mathrm{Si}$ face and from the $\mathrm{C}$ face, then the $6 \mathrm{H} \mathrm{SiC}$ plates again from both sides, and plates $6 \mathrm{H}$ SI SiC were used for measuring of electrical properties. The plates were supplied by Semiconductor Wafer, Inc. Taiwan. The wafers were cut to pieces of $10 \times 10 \mathrm{~mm}$ and cleaned via the standard process [5].

Graphene layers were prepared in the above-mentioned apparatus, under the following conditions: the source of carbon was methane, or mixture of methane and hydrogen at various ratios, preparation temperature $1080^{\circ} \mathrm{C}$, pressure 20 Torr, methane flow $18 \mathrm{sccm}$, growth time 300 $4300 \mathrm{~s}$. The formed layers were analysed by Raman spectroscopy (Thermo Scientific DXR Raman Microscope, $\lambda=532 \mathrm{~nm}, P=7 \mathrm{~mW})$, X-ray Photoelectron Spectroscopy - XPS (Omicron Nanotechnology Ltd.) and basic electrical parameters (resistivity and mobility) were measured by the Van der Paw method.

\section{Results}

Optimization of graphene film preparation was carried out by the CVD growth at constant temperature of $1080^{\circ} \mathrm{C}$ by changing growth time and the ratio of hydrogen and methane flow in the growth chamber. The principal analysis to determine, whether graphene had been prepared on the surface of substrates, was Raman spectroscopy. Figure 1, shows a Raman spectrum of graphene prepared on $4 \mathrm{H} \mathrm{SiC}, \mathrm{Si}$ face, growth time $3600 \mathrm{~s}$, and pure methane spectrum "a". From the spectrum it is evident that the prepared graphene is highly faulty (significant amplitude of the D-peak against the G-peak) and multi-layered (FLG peaks intensity ratio $2 D / G=0.47$ ). This is a graphene film of the best parameters, hence the given conditions of the CVD process can be considered optimal. In numerous cases no $2 \mathrm{D}$ peak occurred at the Raman spectra at all, or just a small one see spectrum "b" at Fig. 1 (graphene prepared under conditions $1100^{\circ}$ C, 20 Torr, $1000 \mathrm{~s}$, gas mixture $\mathrm{H}_{2}: \mathrm{CH}_{4}=1$ : 4). In the course of experiments, influence of the $\mathrm{SiC}$ substrates face was followed as well. It was found that the Si face is more fitting for graphene growth. Quality of prepared graphene was just very slightly dependent on the type of SiC substrate.

*University of Chemistry and Technology, Technická 5, Prague 6, Czech Republic, petr.machac@vscht.cz 


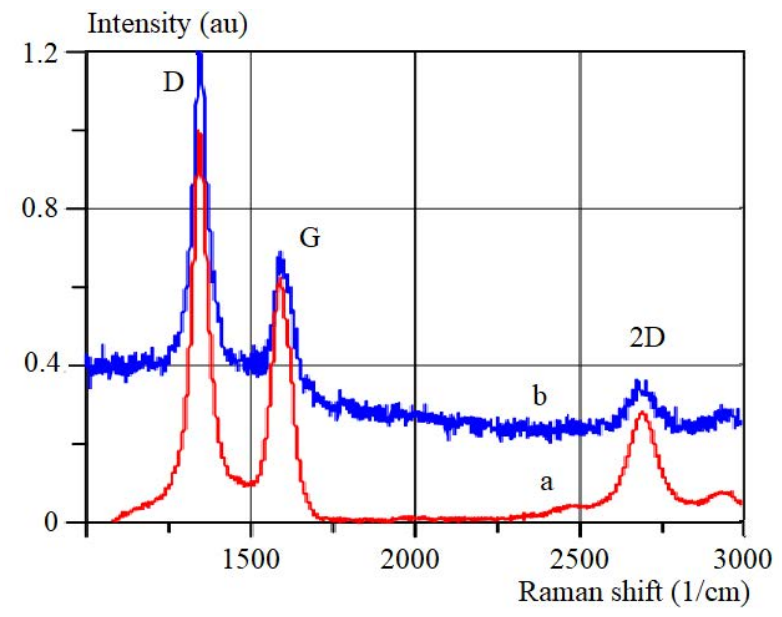

Fig. 1. Raman spectrum of prepared graphene films: "a"- under optimal growth conditions, in atmosphere of pure methane, "b"graphene prepared in gas mixture $\mathrm{H}_{2}: \mathrm{CH}_{4}=1: 4$.

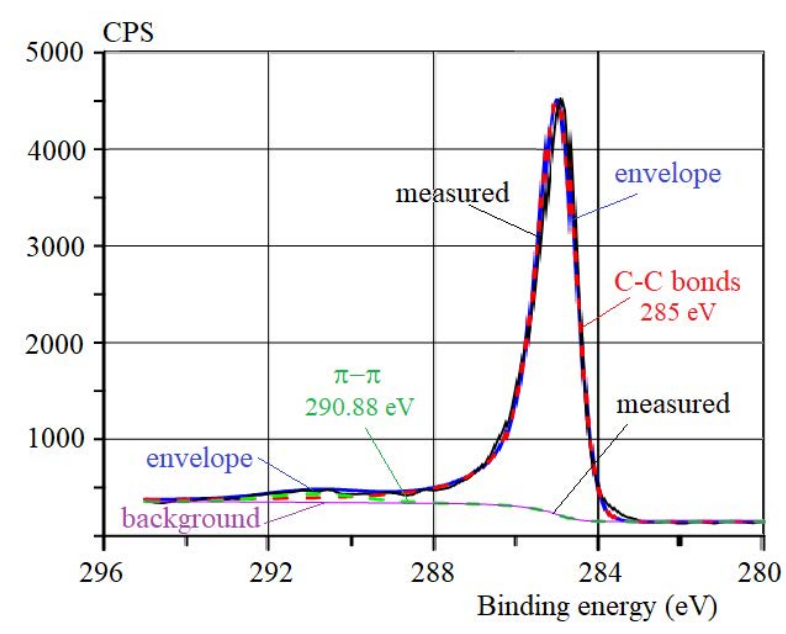

Fig. 3. XPS C 1s core level spectrum. XPS C 1s core level spectrum

For verification of the results of Raman spectroscopy, XPS spectroscopy was used. From the obtained survey spectra Fig. 2, it is evident that only carbon with minimal quantity of oxygen (below one percent) is found on the surface of the samples. Very weak signal of silicon coming from the $\mathrm{SiC}$ substrate is visible in case of electron detection under perpendicular angle. Figure 3 shows detailed XPS spectrum of carbon $\mathrm{C}$ 1s. The black line represents the measured spectrum, the blue line represents spectrum obtained by fitting, when the asymmetric bonding of C-C and $\pi-\pi$ was used (red curve: C-C bonds and green curve: $\pi-\pi$ bonds). Violet curve represents background of the measurement. The band belonging to carbon from $\mathrm{SiC}$ substrate is not present within the spectrum, though there is very weak band corresponding to silicone within the survey spectrum. It is highly important, that the structure contains just a very small quan-

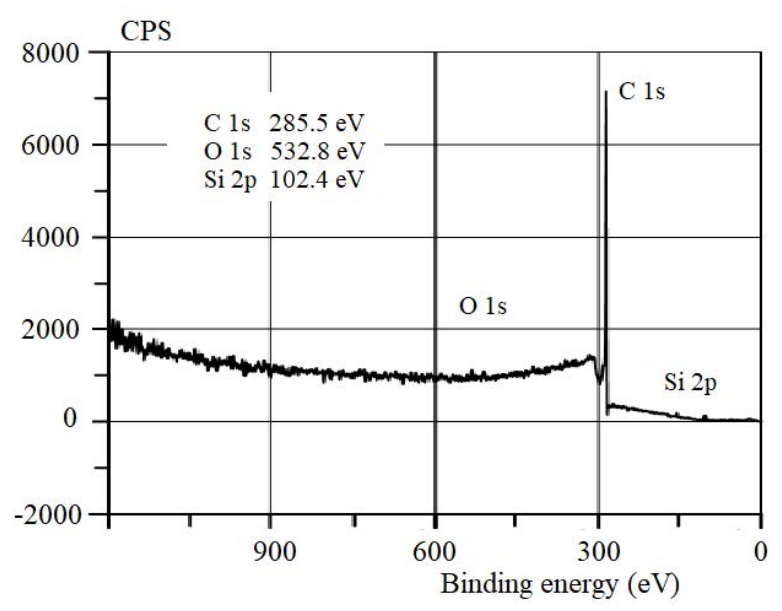

Fig. 2. Survey XPS spectrum measured at perpendicular electron detection

tity of oxygen, which gives evidence that practically no oxidation of graphene film takes place. The shape of Raman spectra of the formed graphene films (Fig. 1) may be nearing to the shape of graphene oxide [6]. The XPS measurement, however, excludes this eventuality; therefore it can be declared that carbon on the surface of $\mathrm{SiC}$ substrates is graphene and not graphene oxide.

Further, electrical properties were observed for the prepared graphene layers. For these measurements, semiinsulating $\mathrm{SiC}$ plate of high resistivity $(6 \mathrm{H} \mathrm{SI} \mathrm{SiC)}$ was used. Rectangular contacts of $\mathrm{Au}(30 \mathrm{~nm}) / \mathrm{Cr}(10 \mathrm{~nm})$ had been prepared for the measurement by vacuum evaporation onto the graphene film in corners of the plates. Surface resistivity was measured at current values 0.1-1 mA, mobility was measured using magnetic field $0.43 \mathrm{~T}$. Values of $\mathrm{R}=1 \mathrm{k} \Omega$ and $\mu=25 \mathrm{~cm}^{2} / \mathrm{Vs}$ are for graphene layers of the highest quality (growth time $3600 \mathrm{~s}$ ).

\section{Conclusions}

For preparation of graphene, the CVD method was used. The substrate for growth was $\mathrm{SiC}$. The given method is similar to that of thermal dissociation of $\mathrm{SiC}$. The source of carbon at CVD are not the carbon atoms that remain at the SiC surface after silicon escape, but carbon atoms originating from dissociated molecules of methane. Both methods have in common the disadvantage of high growth temperature. Using the above-mentioned CVD apparatus, multi-layer graphene films consisting of at least 5 carbon monolayers of relatively high failure rate were prepared at the temperature of $1080^{\circ} \mathrm{C}$. The XPS analysis proved that graphene films show minimal quantity of oxygen. Surface resistance of the layers is $1 \mathrm{k} \Omega$, electron mobility is very low due to high failure rate of the layers, maximal values of $25 \mathrm{~cm}^{2} / \mathrm{Vs}$ were reached. Despite high failure rate, this is 
graphene prepared using the CVD method from methane atmosphere at the lowest temperature among those published in literature. The prepared graphene layers may be used as a base for electronic structures prepared on SiC.

\section{Acknowledgment}

This study was supported by the Czech Science Foundation, Project No. 17-00607S.

\section{REFERENCES}

[1] U. Starke and C. Riedl, "Epitaxial graphene on $\mathrm{SiC}(0001)$ $\mathrm{SiC}(000-1)$ : from surface reconstructions to carbon electronics", J.Phys.Condens.Matter, 21 134016, 2009.

[2] J. G. Kim, W. S. Kim, Y. H. Kim, C. H. Lim, and D. J. Choi, "Formation of graphene on $\mathrm{SiC}$ by chemical vapour deposition with liquid sources", Surface \& Coating Technology, 231,189-192, 2013.

[3] Y. Hagihara, T. Kajiwara, A. Visikovskiy, and S. Tanaka, "Graphene nanoribbons grown on epitaxial $\mathrm{Si}_{x} \mathrm{C}_{y} \mathrm{O}_{z}$ laxer on vicinal $\mathrm{SiC}(0001)$ surfaces by chemical vapour deposition" , Appl.Phys.Express, 6 55102, 2013.

[4] Q. Liu, C. Yu, Z. He, G. Gu, J. Wang, C. Zhou, J. Guo, Y. Gao, and Z. Feng, "Chemical vapour deposition graphene of gradient growth method on an $4 \mathrm{H}-\mathrm{SiC}(0001)$ substrate", Appl.Surf. Sci., 454, 68-73, 2018.

[5] P. Machac, T. Fidler, S. Cichon, and V. Jurka, "Synthesis of graphene on $\mathrm{Co} / \mathrm{SiC}$ structure", J.Mater.Sci.Mater.Electron, 24, 3793-3799, 2013

[6] D. Yang, "Chemical analysis of graphene oxide films after heat chemical treatments by X-ray photoelectron Micro-Raman spectroscopy", Carbon, 47 145-152, 2009.

Received 6 June 2019

Petr Macháč was born in Hranice, Czech Republic, in 1953. In 1977 he graduated in electrical engineering from Czech Technical University Prague and received the $\mathrm{PhD}$ degree in Electronics at the same place, 1982. From 1978 to 1984 he was with Czech Technical University Prague. In 1991 was appointed Associate Professor in Electronics with Czech Technical University Prague, being active with University of Chemistry and Technology, Prague from 1984 by now. His current research interest is in deposition of materials, formation of contacts on semicon-ductor materials (mainly $\mathrm{SiC}$ ), and preparation of graphene layers by several methods. He is the author or co-author of more than 60 articles in scientific journals and 100 contributions on international conferences. 